\title{
Construction of the New Prototype of Main Quadrupole Cold Masses for the Arc Short Straight Sections of LHC
}

\author{
M. Peyrot ${ }^{1}$, J.M. Rifflet ${ }^{1}$, F. Simon ${ }^{1}$, T. Tortschanoff ${ }^{2}$ and P. Vedrine ${ }^{1}$
}

\begin{abstract}
Each cold mass of the short straight sections in the eight LHC arcs will contain a $3.25 \mathrm{~m}$ long twin aperture quadrupole of a nominal gradient of $223 \mathrm{~T} / \mathrm{m}$. This magnet will be aligned in a $5.3 \mathrm{~m}$ long inertia tube together with auxiliary magnets on each end. On the quadrupole connection end either a pair of $38 \mathrm{~cm}$ long octupole or trim quadrupole magnets will be mounted, on the other end there will be combined sextupoledipole correctors with a yoke length of $1.26 \mathrm{~m}$. The powering of the main quadrupoles will be assured by two pairs of copper stabilized superconducting bus-bars placed inside the cold mass next to the bus-bars for the main dipole magnets. Each of the two quadrupole apertures will be connected to its quench protection diode. The construction of three prototypes has been entrusted to the CEA/Saclay laboratory, in the frame of the special French contribution to the LHC project. The first cold mass prototype has been completed and warmmeasured for its multipole content at CEA. The second cold mass is presently under completion. The paper will review the experience with the development of the quadrupole coils and cold mass construction and gives the results of the first warm magnetic measurements. An outlook for the series manufacture of the 400 arc quadrupole magnets and their cold masses for the LHC machine will complete the report.
\end{abstract}

1 CEA/Saclay, France. Members of the CERN-CEA-CNRS Collaboration to the LHC Project

2 CERN, LHC Division

Presented at the 16th International Conference on Magnet Technology

26 September-2 October 1999 - Ponte Vedra Beach, USA

Administrative Secretariat

LHC Division

CERN

CH - 1211 Geneva 23

Switzerland

Geneva, 1 March 2000 


\title{
CONSTRUCTION OF THE NEW PROTOTYPE OF MAIN QUADRUPOLE COLD MASSES FOR THE ARC SHORT STRAIGHT SECTIONS OF LHC
}

\author{
M. Peyrot, J.M. Rifflet, F. Simon, P. Vedrine \\ CEA/Saclay, France \\ T. Tortschanoff \\ CERN, Geneva, Switzerland
}

\begin{abstract}
Each cold mass of the short straight sections in the eight LHC ares will contain a $3.25 \mathrm{~m}$ long twin aperture quadrupole of a nominal gradient of $223 \mathrm{~T} / \mathrm{m}$. This magnet will be aligned in a $5.3 \mathrm{~m}$ long inertia tube together with auxiliary magnets on each end. On the quadrupole connection end either a pair of $38 \mathrm{~cm}$ long octupole or trim quadrupole magnets will be mounted, on the other end there will be combined sextupole-dipole correctors with a yoke length of $1.26 \mathrm{~m}$. The powering of the main quadrupoles will be assured by two pairs of copper stabilized superconducting bus-bars placed inside the cold mass next to the bus-bars for the main dipole magnets. Each of the two quadrupole apertures will be connected to its quench protection diode. The construction of three prototypes has been entrusted to the CEA/Saclay laboratory, in the frame of the special French contribution to the LHC project. The first cold mass prototype has been completed and warm-measured for its multipole content at CEA. The second cold mass is presently under completion. The paper will review the experience with the development of the quadrupole coils and cold mass construction and gives the results of the first warm magnetic measurements. An outlook for the series manufacture of the $\mathbf{4 0 0}$ arc quadrupole magnets and their cold masses for the LHC machine will complete the report.
\end{abstract}

\section{INTRODUCTION}

Since 1989, collaboration between CEA/Saclay and CERN was started for the development of the lattice quadrupole magnets for the LHC. This resulted in the successful construction and testing of two prototype quadrupole magnets operated in the LHC Test String between 1994 and 1998 [1]. Following the approval of the LHC project, a new co-operative program to develop quadrupole magnets has been established between CEA/Saclay and CERN as part of the special French participation to the LHC accelerator project being carried out at CERN. CEA/Saclay will provide three cold masses of the short straight section including the $3.25 \mathrm{~m}$ long twin aperture lattice quadrupole, combined dipole-sextupole corrector magnets on one end of the quadrupole and on the other end a pair of octupole or tuning quadrupole corrector magnets. The short straight section units are fully described in [2]. The quadrupole cross-section is shown in Fig. 1. This paper presents the construction of the lattice quadrupole magnet and its assembly with corrector magnets

Manuscript submitted September 27, 1999 to complete the first $5.5 \mathrm{~m}$ long cold mass prototype called SSS3.

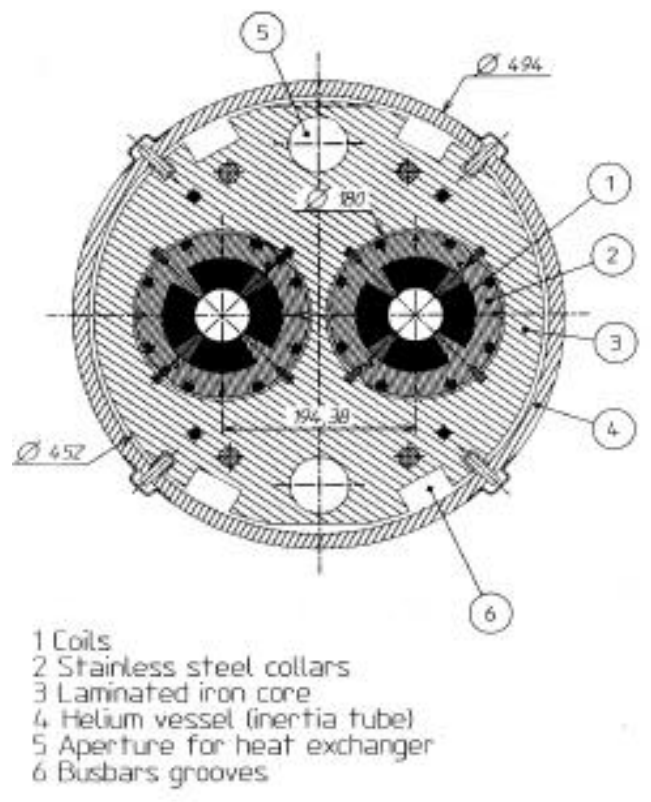

Fig. 1. Cold mass cross section of the LHC short straight section

\section{MAGNET DESIGN}

Each of the four coils per aperture consists of 10 turns in the inner layer and 14 turns in the outer, with three copper wedges, one in the inner layer and two in the outer. Both layers are made of the same keystoned Rutherford-type superconducting cable without splice. The cable is the same as the one used for the second layer of the coil of the LHC dipole. It consists of $360.825 \mathrm{~mm}$ strands and has a crosssection with a minor edge of $1.362 \mathrm{~mm}$, major edge of $1.598 \mathrm{~mm}$ and width of $15.1 \mathrm{~mm}$. The $\mathrm{Nb}$-Ti filament size is $6 \mu \mathrm{m}$, and the $\mathrm{Cu}: \mathrm{Sc}$ ratio is 1.9 . The cable critical current at $9 \mathrm{~T}$ and $1.9 \mathrm{~K}$ is greater than $12950 \mathrm{~A}$. The cable is insulated using three wraps. The first wrap (inner wrap) is made of Kapton ${ }^{\mathrm{TM}}$ film $50 \mu \mathrm{m}$ thick and $11 \mathrm{~mm}$ wide wound edge-to-edge in the reverse direction of that of the cable. The second wrap is made of Kapton ${ }^{\mathrm{TM}}$ film $37 \mu \mathrm{m}$ thick and $11 \mathrm{~mm}$ wide wound edge-to-edge in the same direction as the first wrap but shifted by $5.5 \mathrm{~mm}$ from the first one. The third wrap is made of Kapton ${ }^{\mathrm{TM}}$ film $50 \mu \mathrm{m}$ (plus $5 \mu \mathrm{m}$ of polyimide adhesive) thick wound with a $2 \mathrm{~mm}$ spacing. The prestress of the arc quadrupole coils is 
provided by $2.5 \mathrm{~mm}$ thick laminated stainless steel collars, with a target coil prestress of $80 \mathrm{MPa}$ at $300 \mathrm{~K}$. The collars are locked by a set of eight tapered keys regularly located around the circumference. This structure withstands the electromagnetic forces without contribution of the yoke. The two collared apertures are centred inside the single piece yoke laminations by longitudinal keys. The centring of the yoke inside the inertia tube is again provided by a set of keys that are blocked from the outside of the tube by a series of bolts. A pair of octupole or trim quadrupole corrector magnets is placed at the connection end of the quadrupole. At the other end is located a combined twin aperture dipolesextupole corrector magnet. Both corrector magnets are located and aligned inside the inertia tube in the same way as the quadrupole. The inertia tube serves as helium vessel and encloses also three pairs of bus-bars held in grooves outside of the quadrupole yoke; one pair passes through the cold mass for powering adjacent dipoles and two pairs for the powering of the focusing and defocusing quadrupole. For protecting the magnets against the damage caused by quenches, each aperture is connected by dedicated bus-bars to a current bypassing diode. These diodes are housed in a stainless steel can attached to the connection side end cover of the cold mass. About one fourth of the cold masses will need additional equipment. For respecting the separation of the coolant circuits of the machine, the tubes guiding the bus-bars to the neighboring magnet on the connection side must be equipped with pressure plugs. Furthermore, for sectoring the machine cryostat insulation vacuum, about every fourth cold mass must be equipped with an interface ring for the vacuum barrier welded around the outside of the inertia tube.

Table 1: Main parameters and characteristics of the LHC quadrupole magnet and cold mass

\begin{tabular}{lcc}
\hline $\begin{array}{l}\text { Injection field gradient (0.45 TeV beam } \\
\text { energy) }\end{array}$ & Value & Unit \\
\hline $\begin{array}{l}\text { Nominal field gradient (7 TeV beam } \\
\text { energy) }\end{array}$ & 223 & $\mathrm{~T} / \mathrm{m}$ \\
\hline Nominal current & $11^{\prime} 870$ & $\mathrm{~T} / \mathrm{m}$ \\
\hline Operating temperature & 1.9 & $\mathrm{~K}$ \\
\hline Magnetic length at 1.9 K & 3.1 & $\mathrm{~m}$ \\
\hline Stored energy (both apertures) at 7 TeV & 0.79 & $\mathrm{MJ}$ \\
\hline Ultimate operational field gradient & 241 & $\mathrm{~T} / \mathrm{m}$ \\
\hline Gradient at short sample field limit & 278 & $\mathrm{~T} / \mathrm{m}$ \\
\hline Distance between aperture axis at 1.9 K & 194.00 & $\mathrm{~mm}$ \\
\hline Inner coil diameter at 293 K & 56.00 & $\mathrm{~mm}$ \\
\hline Outer coil diameter at 293 K & 118.60 & $\mathrm{~mm}$ \\
\hline Number of conductor blocks / pole & 4 & \\
\hline $\begin{array}{l}\text { Electromagnetic forces / coil octant at } \\
\text { nominal field }\end{array}$ & & \\
$\begin{array}{l}\text { Radial force (inner and outer layer) } \\
\text { Azimuthal force (inner and outer layer) }\end{array}$ & 0.54 & $\mathrm{MN} / \mathrm{m}$ \\
\hline $\begin{array}{l}\text { Cold mass length at 293 K, between end } \\
\text { covers }\end{array}$ & 5355 & $\mathrm{MN} / \mathrm{m}$ \\
\hline Cold mass overall length with ancillaries & 6.63 & $\mathrm{~mm}$ \\
\hline Cold mass weight & 6.5 & $\mathrm{~T}$ \\
\hline
\end{tabular}

\section{QUADRUPOLE FABRICATION}

\section{A. Coil}

The azimuthal size of the coils determines the collared coil prestress. This nominal size is achieved by the accuracy of the azimuthal dimensions of the mandrel/mould combination cavity during curing. These dimensions were previously tuned by a number of ten-stack measurements. More than 20 coils with G-11 end spacers have been wound, cured and measured. The RMS spread of azimuthal size for each coil is within $\pm 10 \mu \mathrm{m}$, and the difference in the average coil size of the eight coils, from four different cable batches, used for the first completed prototype was $\pm 20 \mu \mathrm{m}$. Typical measurements of the E-modulus for increasing stress is between 7 and $8 \mathrm{GPa}$. On the first eight coils, slight displacements toward aperture of the last conductor block of the first layer were discovered after collaring. As these displacements limit the nominal aperture of the magnet, it was decided to impregnate the coil ends with a heavily charged (about $70 \%$ in weight) epoxy resin system.

\section{B. Collared coil}

In order to finely determine the azimuthal dimension of the mandrel/mould cavity and to complete the ten stack compressive tests, two $200 \mathrm{~mm}$-long models (see Fig. 2) were assembled and collared with 8 capacitive gauges [3].

These gauges are located on a single section of the model, in order to monitor the azimuthal stress applied on the two layers of the coils during collaring and cool-down to $77 \mathrm{~K}$. They are inserted into dedicated grooves machined inside protection sheets located between coils and pole piece of the collars. The two short models are assembled with coils, which have slight changes in their azimuthal dimensions.

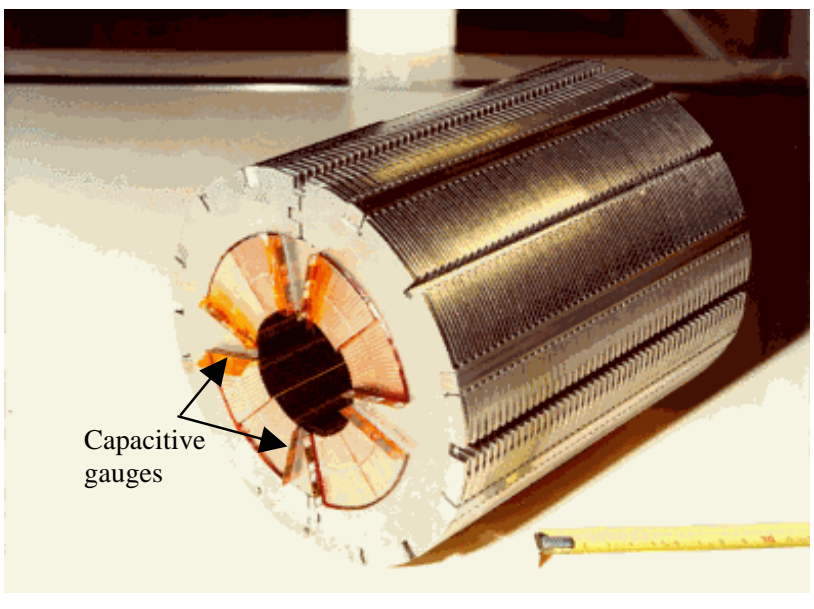

Fig. 2. The short model of prototype quadrupole magnet with capacitive stress gauges 
The mean prestress applied on the coils of the first model was $96 \mathrm{MPa}$ with a standard deviation of $14 \mathrm{MPa}$ for a prestress target of $80 \mathrm{MPa}$. The second short model was assembled with a coil cured with a reduced mandrel/mould cavity. The mean prestress achieved was then $88 \mathrm{MPa}$ with a standard deviation of $5.5 \mathrm{MPa}$. The differences of stress between the two layers of the coils in both models stay within measurement errors and are negligible.After assembly, the short models were cooled down to liquid nitrogen temperature. The loss of prestress was $23 \mathrm{MPa}$ for the first coil and $18 \mathrm{MPa}$ for the second coil, according to previous calculations. Four coils were assembled on an expandable mandrel in the horizontal position and equipped with ground insulation, quench protection heaters and stainless steel protection sheets equipped with capacitive gauges. Once put in the vertical position the collars are then individually stacked. The tapered keys are inserted with sliding strips by means of a $200 \mathrm{~mm}$-long collaring press. The design of the collar/tapered keys combination allows a collaring without overstress inside the coils.

\section{Twin aperture quadrupole magnet}

The two collared coils are mounted in a common yoke made of single-piece low-carbon steel laminations. They are centred in the yoke by means of four keys, which define precisely two orthogonal planes. The stacked laminations are pressed between two stainless steel end plates by means of four tie rods. Fig. 3 shows a quadrupole magnet completed and ready to be assembled into its cold mass.

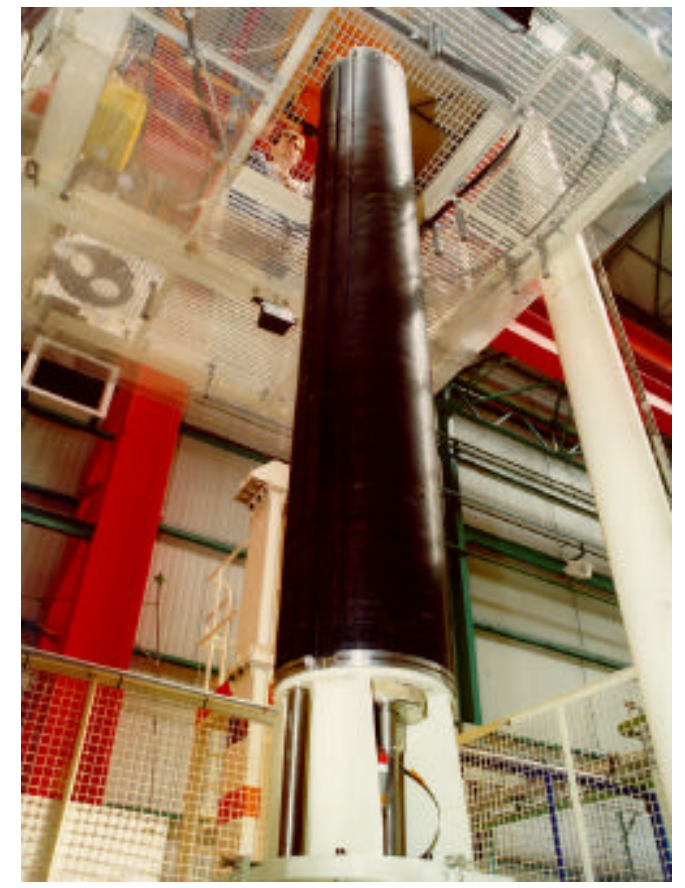

Fig. 3. Twin aperture quadrupole magnet completed

\section{Cold Mass Assembly}

The assembly of the cold mass [4] starts with the lowering of the quadrupole magnet on a dedicated tooling, on which either a pair of octupole magnets or tuning quadrupole magnets has been previously installed. A combined dipole-sextupole is then placed on top of the quadrupole and the whole assembly is equipped with three sets of bus-bars. The connecting of the quadrupole magnet to the bus-bars takes place around the octupole corrector magnets. Fig. 4 shows these completed connections while Fig. 5 shows the combined dipole-sextupole on top of the quadrupole with the two sets of bus-bars. Centring keys are inserted in four grooves all along this assembly and the inertia tube is lowered around it. The keys are then extracted from the outside to the inner diameter of the tube in order to centre and align the magnets. Once in the horizontal position the cold mass is closed by two stainless steel end covers. It is pressure and leak tested and ready for warm magnetic measurements.

\section{Warm Magnetic Measurements}

The magnets has been measured with a mole-type device developped with CERN. It consists of a rotating array of 5 radial coils. Each 200-turn coil were wound at CERN on $750 \mathrm{~mm}$ long and precisely machined mandrels. The coils were calibrated in a dipole and a quadrupole to determine the size and position of the coils. This allows to make a hardware bucking of about 900 for the dipole and 400 for quadrupole terms.

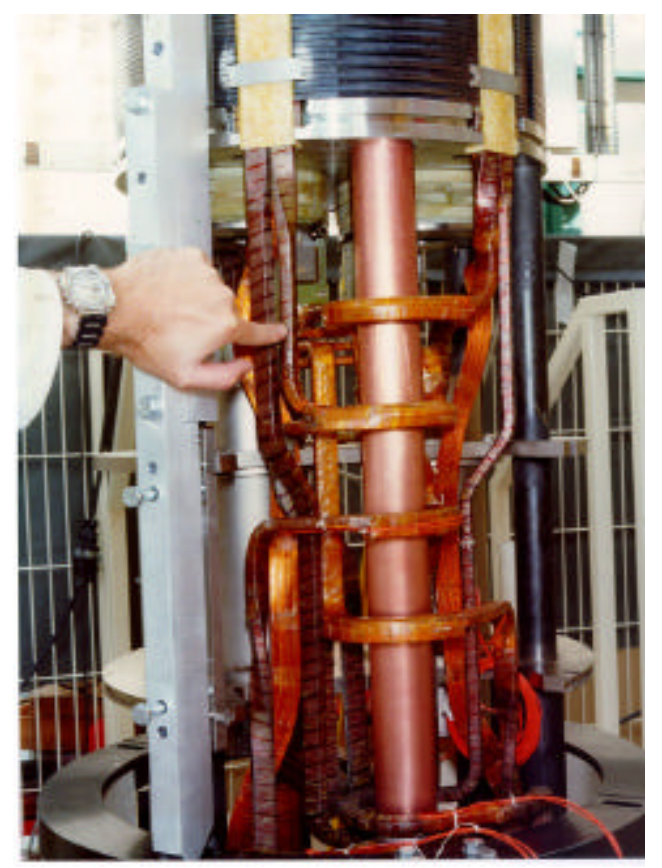

Fig. 4. View of the connections of the quadrupole to the bus-bars around the octupoles and heat exchanger tube. 


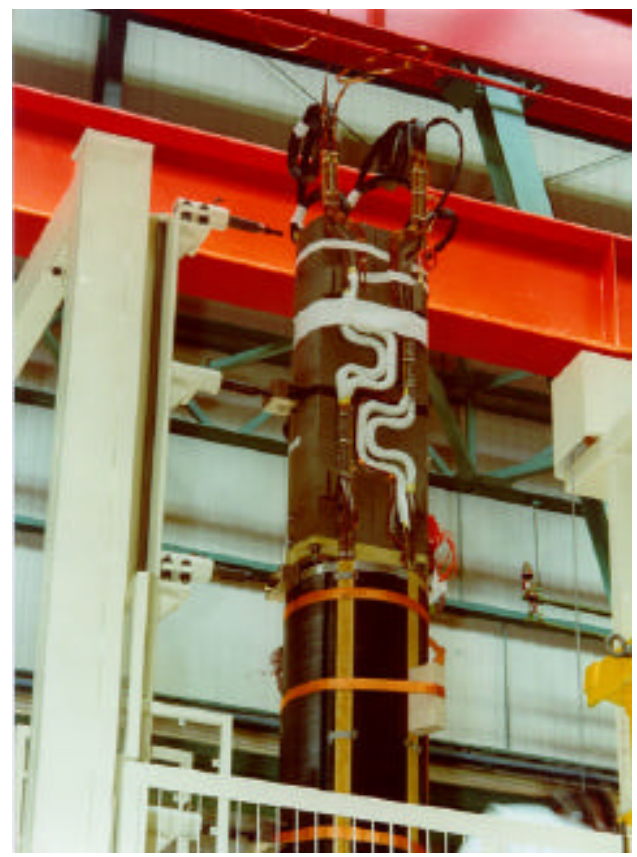

Fig. 5. View of the top of the assembly before lowering of the inertia tube.

The reproductibility is a few $10^{-6}$ with respect to $B_{2}[5]$. This system was compared with another system used in CEA for cold test. The two systems give the same results on a quadrupole up to the $b_{6}$. Up to now, we have measured (see Table 2) the two apertures of the cold mass and its two collared coils before yoking at warm temperature. The sextupole and dodecapole coefficients are high but within the required limits. The gradient would be $221.6 \mathrm{~T} / \mathrm{m}$ at the nominal current with the yoke and the magnetic length $3114 \mathrm{~mm}$ when $223.05 \mathrm{~T} / \mathrm{m}$ and $3112 \mathrm{~mm}$ are expected. More coils are needed to do a statistic, detect trends and correct possible imperfections.

\section{INDUSTRIALISATION}

After the completion and the test of the two next cold mass prototypes, industrial production will start. A market survey was launched to qualify firms, to which the call for tender will be sent. These firms were invited to assist at the critical stages on the prototype fabrication at CEA/Saclay during 1999.

The call for tender will be sent out during the following month, in order to have the contract placed in the first half of 2000. To avoid the risk of failure and to achieve a good production rate, it is foreseen to have two firms in charge of the fabrication of the cold masses. CEA/Saclay will transmit a fully detailed technical documentation and will send the persons who built the prototypes to the firms in order that they transmit their knowledge of technology.
Table 2: Integrated multipole coefficients in unit $\left(10^{-4} \mathrm{~B}_{2}\right)$ at warm temperature and $17 \mathrm{~mm}$ radius.

\begin{tabular}{lcccc}
\hline & \multicolumn{2}{c}{ External left coil } & \multicolumn{2}{c}{ Internal right coil } \\
\cline { 2 - 5 } & collared & Yoked & collared & yoked \\
\hline $\mathrm{b}_{3}$ & 3.86 & 3.20 & 1.89 & 1.88 \\
\hline $\mathrm{b}_{4}$ & -0.09 & -0.18 & 0.08 & 0.06 \\
\hline $\mathrm{b}_{6}$ & 5.99 & 5.59 & 6.67 & 6.25 \\
\hline $\mathrm{b}_{10}$ & -0.12 & -0.12 & -0.12 & -0.12 \\
\hline $\mathrm{a}_{3}$ & -3.38 & -3.19 & -0.53 & -0.42 \\
\hline $\mathrm{a}_{4}$ & 2.48 & 2.42 & 1.03 & 0.89 \\
\hline $\mathrm{a}_{6}$ & 0.16 & 0.61 & 0.08 & 0.58 \\
\hline $\mathrm{a}_{10}$ & 0.05 & 0.04 & 0.03 & 0.01 \\
\hline
\end{tabular}

The technical documentation will contain the description and the drawings of the tooling and components, detailed assembly procedures and the quality assurance plan used for the prototypes including control and test procedures.As CEA/Saclay will also be in charge of the follow-up of preseries and series fabrication, CEA/Saclay's staff will be present at the firms. However, firms will be fully responsible for the supply of tooling and components and of their fabrication. Some material (superconducting cable, polyimide insulating tape and sheets, bus-bars, corrector magnets, diode assembly, steel, ...) will be supplied by CERN from within separate contracts.

\section{CONCLUSIONS}

The first new prototype, SSS3, has been completed at CEA/Saclay and sent to CERN. It will be put in a cryostat and cold tested at CERN in the next few months. Quality assurance plan and procedures are ready for industrialization and will be finalized during the fabrication of the next two cold masses. The latter are currently under completion. They will be cold-tested at CEA/Saclay next year before being sent to CERN for cryostating. The three prototype cold masses are fully instrumented to allow detailed analyses of their performances.

\section{REFERENCES}

[1] A. Bézaguet et al., "The LHC Test String: First Operational Experience", Proc. EPAC96 Conference, Sitges, Barcelona, Spain. 1996.

[2] T. Tortschanoff et al., "The Short Straight Sections For the LHC", Proc. PAC97 Conference, Vancouver, Canada. 1997.

[3] L. Vieillard et al, "Capacitive Force sensors for Prestress in Accelerator magnet coils", Proc. CEC99 Conference, Montréal, Canada. 1999.

[4] M. Gagné et al., "Study of Short Straight Section Cold Mass Assembly Principles - Presentation of the Assembly Process Resulting from this Study", CERN-LHC-Project Note 73. 1996.

[5] A. Devred et al., "Dynamic Field Quality of LHC / Saclay Arc Quadrupole Magnet Prototype", Proc. PAC97 Conference, Vancouver, Canada. 1997. 